\title{
Sliding Mode Control with System Constraints for Aircraft Engines
}

\author{
Shu-Bo Yang ${ }^{\mathrm{a}, \mathrm{b}}$, Xi Wang ${ }^{\mathrm{a}, \mathrm{b}, *}$, Hao-Nan Wang ${ }^{\mathrm{a}, \mathrm{b}}$, Yi-Guang $\mathrm{Li}^{\mathrm{c}}$ \\ ${ }^{a}$ Collaborative Innovation Center for Advanced Aero-Engine, 100191 Beijing, China \\ ${ }^{b}$ School of Energy and Power Engineering, Beihang University, 100191 Beijing, China \\ ${ }^{c}$ Cranfield University, Bedford MK43 0AL, United Kingdom
}

\begin{abstract}
This paper proposes a constraint-tolerant design with sliding mode strategy to improve the stability of aircraft engine control. To handle the difficulties associated with the high-frequency switching laws, merely attenuating the chattering is far from satisfactory. System constraints on input, output, and input rate should be addressed in the design process. For a sort of uncertain nonlinear systems subjected to the constraints, sliding mode regulators are designed using Lyapunov analysis. A turbofan engine is adopted for simulation, which shows that the methodology developed in this paper can handle the speed tracking and limit protection problem in a stable fashion, despite the negative influence posed by the system constraints.
\end{abstract}

Keywords: aircraft engine control, speed tracking, limit protection, sliding mode control, system constraints, system uncertainties, turbofan engine

\section{Introduction}

With years of development, aircraft engines require a complex control system to achieve both performance and protection objectives. The control system is generally employed for realising desired steady-state and transient performance, as well as limit protection[1]. Fig. 1 shows the most common framework(the max-min switching framework) in the aircraft engine control field, which includes all those three functions. The main advantage of this framework is that the original tasks are decoupled into subtasks, which then can be dealt with separately.

To build such a control system, different control methods such as PID control, sliding mode control (SMC), adaptive control and so on may be used. Among these methods, SMC is suitable for limit protection because of its monotone convergence property. Due to its mature theories and numerous successful applications $[2,3,4,5]$, SMC has been considered in aircraft engine control. For instance, Ritcher suggests replacing linear regulators with sliding ones. Comparing with the standard max-min approach, this method has less conservation and can improve the performance of the whole system. He demonstrated in his work[6] that SMC with monotone convergence property can guarantee that all system outputs will not exceed their limits and further can keep small limit margin in exchange for better performance.

In aircraft engine control, except for output constraints being introduced deliberately as limit protections[7], input and input rate constraints naturally exist in closed-loop systems. For some actuators such as fuel metering valve, they have their own limits on position and motion, which may lead to magnitude or rate saturation during their control process[8]. Because of the high-frequency switching laws, it is not surprising that SMC has difficulties in compliance with mechanical constraints, especially the rate constraints. To handle the difficulties, numerous studies have been done on chattering suppression[9, 10, 11, 12, 13]. Introducing a sigmoid function[9] or other continuous functions[10] as the boundary layer is a simple and practical technique. Alternatively, higher-order SMC[11] and the reaching law approach[12, 13] can be applied to achieve

\footnotetext{
* Corresponding author

Email address: xwang@buaa.edu.cn (Xi Wang)
} 


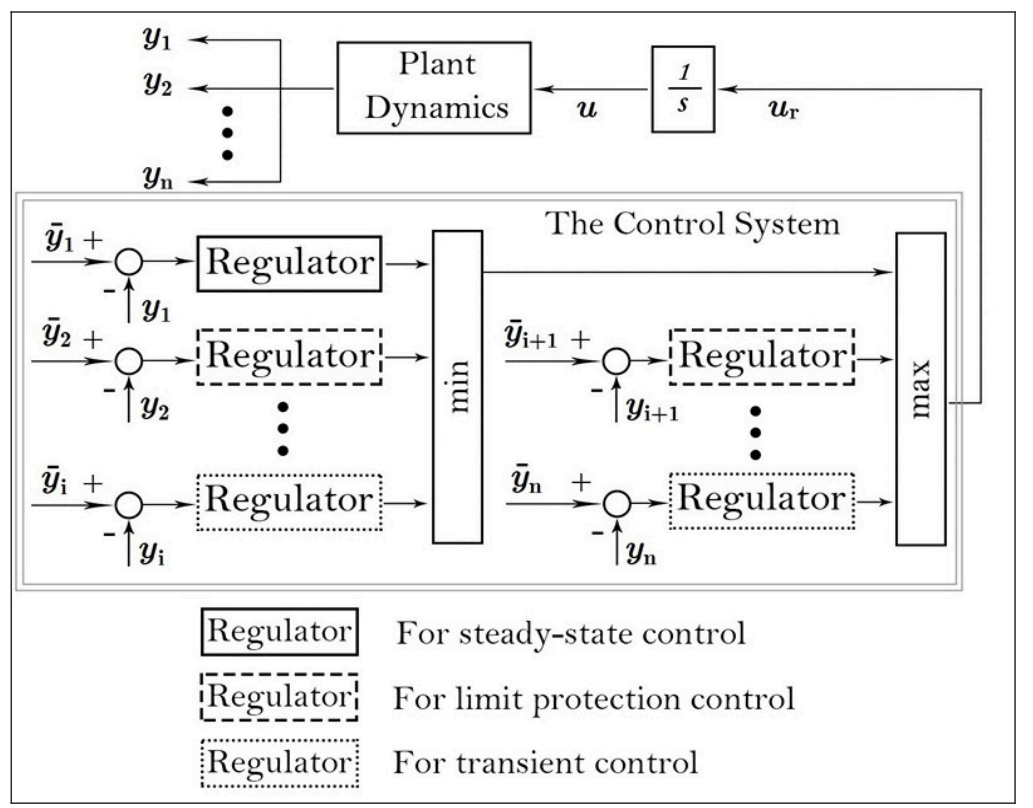

Figure 1: Commonly used switching framework for aircraft engine control.

better performance. However, it is found that no matter what methods are employed, only attenuating the chattering is far from satisfactory. Because a chattering-free control still cannot guarantee that the rate of control will not exceed its limit during the reaching phase. Once reaching the border of its rate constraint, an actuator will be saturated and may not execute the actual command from the control system, which may severely degrade the system performance or even endanger the system safety.

Since merely attenuating the chattering has limited ability to improve the closed-loop performance, this paper aims at proposing a new constraint-tolerant design to address system constraints in the design process. The main contributions are listed as follows:

a) to handle the difficulties associated with the high-frequency switching laws, instead of chattering suppression, three types of system constraints are addressed in the design process.

b) for a sort of uncertain nonlinear systems subjected to the constraints, the structure of sliding mode regulators is improved and corresponding stability analysis is carried out.

The rest of this paper is organised as follows: In section 2, the control problem will be formulated. In section 3 , with the aid of $[14,15,16]$, a general sliding function is defined for both main regulators and limit regulators, and an SMC law is synthesised with thorough stability analysis. In section 4, the proposed design is applied to a reliable model turbofan. In section 5, a conclusion of the paper is provided.

\section{Problem formulation}

\subsection{Overall description}

Consider a nonlinear dynamical system represented by Eq. (1)[17].

$$
\begin{aligned}
& \dot{\mathbf{x}}(t)=f(\mathbf{x}(t), u(t)) \\
& \mathbf{y}(t)=g(\mathbf{x}(t), u(t))
\end{aligned}
$$


where $u(t) \in \Re$ is the input to the engine, $\mathbf{x}(t) \in \Re^{n}$ is the vector of engine state, $\mathbf{y}(t) \in \Re^{m}$ is the engine outputs, $f(\cdot)$ and $g(\cdot)$ are differentiable nonlinear functions with n-dimension and m-dimension respectively. The former function represents the dynamics of the engine while the latter one generates the outputs of the engine.

The control objective is to solve a tracking problem under specific constraints. Without loss of generality, assume $y_{1}(t)$ to be the output of steady-state control and $y_{i}(t)$, where $i=2, \ldots, m$, be the outputs for limit protection or transient control. To generate a control system so that $y_{1}(t)$ tracks a reference signal as time goes to infinity, the control process must satisfy the output, input, and input rate constraints represented by Eq. (2).

$$
\begin{gathered}
y_{i}^{L} \leq y_{i}(t) \leq y_{i}^{U} \\
u^{L} \leq u(t) \leq u^{U} \\
\dot{u}^{L} \leq \dot{u}(t) \leq \dot{u}^{U}
\end{gathered}
$$

where superscript "L" denotes the lower bound while "U" denotes the upper bound.

For any possible output $\mathbf{r} \in \mathbb{D} \subset \Re^{m}$, where $\mathbb{D}$ is a compact set, there exists a unique pair $\left(\mathbf{x}_{e}, u_{e}\right)$ which satisfies Eq. (3)[17].

$$
\begin{aligned}
& f\left(\mathbf{x}_{e}, u_{e}\right)=\dot{\mathbf{x}}_{e}=0 \\
& g\left(\mathbf{x}_{e}, u_{e}\right)=\mathbf{y}_{e}=\mathbf{r}
\end{aligned}
$$

where $\mathbf{x}_{e}$ and $u_{e}$ are engine state and control input of the corresponding equilibrium operating point.

\subsection{Local linear-based description}

A thermodynamic aero-engine model cannot be expressed explicitly due to its complexity. Generally, most of the commercial software used for gas turbine modeling and simulation resort to a linearisation method $[18,19,20]$. Therefore, it is reasonable to linearise the plant represented by Eq. (1) at a set of chosen equilibrium points. Without loss of generality, $\left(\overline{\mathbf{x}}_{e}, \bar{u}_{e}\right)$ is used to denote any one of the chosen equilibrium points.

The results of the linearisation at $\left(\overline{\mathbf{x}}_{e}, \bar{u}_{e}\right)$ can be expressed as

$$
\begin{aligned}
\delta \dot{\mathbf{x}}(t) & =\mathbf{A} \cdot \delta \mathbf{x}(t)+\mathbf{B} \cdot \delta u(t) \\
\delta y_{i}(t) & =\mathbf{C}_{i} \cdot \delta \mathbf{x}(t)+\mathbf{D}_{i} \cdot \delta u(t)
\end{aligned}
$$

where $i=1, \ldots, m, \delta u(t)=u(t)-\bar{u}_{e}, \delta \mathbf{x}(t)=\mathbf{x}(t)-\overline{\mathbf{x}}_{e}, \delta y_{i}(t)=y_{i}(t)-\bar{y}_{e i} . \mathbf{A}, \mathbf{B}, \mathbf{C}_{i}, \mathbf{D}_{i}$ are the matrices with appropriate dimensions.

It can be seen that when the control input $u(t)$ exceeds its constraints, the plant will have a constant input. Therefore, two scenaria may be discussed as follows.

- Case A : $u(t)$ is not saturated

If the control input $u(t)$ is not saturated, we have $u^{L}<u(t)<u^{U}$. Combined with the input rate constraints and uncertainties, the system represented by Eq. (4) can be rewritten as Eq. (5).

$$
\begin{aligned}
\delta \dot{\mathbf{x}}(t) & =[\mathbf{A}+\Delta \mathbf{A}(t)] \delta \mathbf{x}(t)+[\mathbf{B}+\Delta \mathbf{B}(t)] \delta u(t) \\
\delta \dot{u}(t) & =\operatorname{sat}\left(u_{r}(t)\right) \\
\delta y_{i}(t) & =\mathbf{C}_{i} \cdot \delta \mathbf{x}(t)+\mathbf{D}_{i} \cdot \delta u(t)+w_{i}(t)
\end{aligned}
$$

where scalar $u_{r}(t)$ represents the control input rate. $\Delta \mathbf{A}(t), \Delta \mathbf{B}(t)$ represent state and input uncertainties respectively, $w_{i}(t)$ represents the uncertainty of the i-th output. For all $u_{r} \in \Re$, sat $(\cdot)$ is the saturation function given by

$$
\operatorname{sat}\left(u_{r}\right)= \begin{cases}u_{r} & \text { if }\left|u_{r}\right|<k_{A} \\ k_{A} \cdot \operatorname{sgn}\left(u_{r}\right) & \text { if }\left|u_{r}\right| \geq k_{A}\end{cases}
$$

where $k_{A}>0$. 
- Case B : $u(t)$ is saturated

If the control input is saturated, the following dynamics with the constraints and the uncertainties can be produced.

$$
\begin{aligned}
\delta \dot{\mathbf{x}}(t) & =[\mathbf{A}+\Delta \mathbf{A}(t)] \delta \mathbf{x}(t)+[\mathbf{B}+\Delta \mathbf{B}(t)] \delta \bar{u} \\
\delta y_{i}(t) & =\mathbf{C}_{i} \cdot \delta \mathbf{x}(t)+\mathbf{D}_{i} \cdot \delta \bar{u}+\bar{w}_{i}(t)
\end{aligned}
$$

where

$$
\delta \bar{u}= \begin{cases}u^{U}-\bar{u}_{e} & \text { if } u(t) \geq u^{U} \\ u^{L}-\bar{u}_{e} & \text { if } u(t) \leq u^{L}\end{cases}
$$

Remark 1. In Case B, the control input of the system (7) is always a constant, which means that the stability of the system depends on its own property. Fortunately, it is well-known that all gas turbine systems are self-stable. Therefore, the system (7) with a constant $\delta \bar{u}$ will always be stable.

Some assumptions for system uncertainties $\Delta \mathbf{A}(t), \Delta \mathbf{B}(t)$ and $w_{i}(t)$ are made as below.

Assumption 1. [21, 22] The matrices $\Delta \mathbf{A}(t)$ and $\Delta \mathbf{B}(t)$ represent the uncertainty and satisfy

$$
[\Delta \mathbf{A}(t) \quad \Delta \mathbf{B}(t)]=\mathbf{E} \cdot\left[\begin{array}{ll}
\mathbf{F}_{a}(t) \cdot \mathbf{H}_{a} & \mathbf{F}_{b}(t) \cdot \mathbf{H}_{b}
\end{array}\right]
$$

where $\mathbf{E}, \mathbf{H}_{a}$ and $\mathbf{H}_{b}$ are known constant matrices, while $\mathbf{F}_{a}(t)$ and $\mathbf{F}_{b}(t)$ are unknown time-varying matrices satisfying $\mathbf{F}_{a}^{T}(t) \mathbf{F}_{a}(t) \leq \mathbf{I}$ and $\mathbf{F}_{b}^{T}(t) \mathbf{F}_{b}(t) \leq \mathbf{I}$.

Assumption 2. [21] All output uncertainties $w_{i}(t) \in \Re$ are bounded and can be presented as

$$
w_{i}(t)=\Delta \mathbf{C}_{i}(t) \cdot \delta \mathbf{x}(t)+\Delta \mathbf{D}_{i}(t) \cdot \delta u(t)
$$

where $\Delta \mathbf{C}_{i}(t)$ and $\Delta \mathbf{D}_{i}(t)$ are bounded matrices. Moreover, $w_{i}(t)$ is differentiable and

$$
\left|\dot{w}_{i}(t)\right| \leq k_{B}, \forall t \geq 0
$$

where $k_{B}$ is a constant.

Remark 2. Since the system uncertainties are introduced to represent the mismatch between the real engine and the linearised model, it is reasonable to assume a bounded mismatch in Assumptions 1 and 2.

Definition 1. [23] For all $u_{r} \in \Re$, a function is defined as $\omega(\cdot)$ to denote the excess degree of the boundary $k_{A}$.

$$
\omega\left(u_{r}\right)=\operatorname{sat}\left(u_{r}\right) / u_{r}= \begin{cases}1 & \text { if }\left|u_{r}\right|<k_{A} \\ k_{A} \cdot \operatorname{sgn}\left(u_{r}\right) / u_{r} & \text { if }\left|u_{r}\right| \geq k_{A}\end{cases}
$$

Remark 3. When $u_{r}$ is within the boundary, then $\omega\left(u_{r}\right)=1$. Otherwise, $\omega\left(u_{r}\right)>0$ reduces with the excess degree increasing. As a result, a positive number $\eta$ always exists satisfying

$$
0<\eta<\omega\left(u_{r}\right) \leq 1
$$

\section{Controller design}

According to Remark 1, only the situation where $u^{L}<u(t)<u^{U}$ is discussed in this paper. We will first present the design process of a set of regulators in Section 3.1, and then discuss the solution with some additional considerations in Section 3.2. 
Table 1: Expressions of $\boldsymbol{\Psi}_{i}(t), \Theta_{i}(t)$ and $\Gamma_{i}(t)$

\begin{tabular}{lll}
\hline Variable & Case 1: & Case 2: \\
& $\mathbf{D}_{i}+\Delta \mathbf{D}_{i}(t)=0$ & $\mathbf{D}_{i}+\Delta \mathbf{D}_{i}(t) \neq 0$ \\
\hline $\mathbf{\Psi}_{i}(t)$ & $\mathbf{G}_{i}$ & $\mathbf{C}_{i}+\Delta \mathbf{C}_{i}(t)$ \\
$\Theta_{i}(t)$ & 1 & $\mathbf{D}_{i}+\Delta \mathbf{D}_{i}(t)$ \\
$\Gamma_{i}(t)$ & $\mathbf{G}_{i} \cdot \delta \mathbf{x}_{e}(t)+\delta u_{e}(t)$ & $\delta r_{i}(t)$ \\
\hline
\end{tabular}

\subsection{Design for a set of SMC regulators}

Consider a set of sliding functions

$$
s_{i}(t)=\mathbf{\Psi}_{i}(t) \cdot \delta \mathbf{x}(t)+\Theta_{i}(t) \cdot \delta u(t)-\Gamma_{i}(t)
$$

The expressions of $\boldsymbol{\Psi}_{i}(t), \Theta_{i}(t)$ and $\Gamma_{i}(t)$ are shown in Tab. 1, where $\delta r_{i}(t)=r_{i}(t)-\bar{y}_{e i}, \mathbf{G}_{i}$ is a matrix comprising of the sliding coefficients which can be manipulated, $\delta \mathbf{x}_{e}(t)=\mathbf{x}_{e}(t)-\overline{\mathbf{x}}_{e}, \delta u_{e}(t)=u_{e}(t)-\bar{u}_{e}$, and the pair $\left(\mathbf{x}_{e}, u_{e}\right)$ depends on a provided $\mathbf{r}$, presented in Eq. (3).

Remark 4. It is a top priority to choose the control error to be sliding functions, especially for a tracking task.

$$
\begin{aligned}
s_{i}(t) & =\delta y_{i}(t)-\delta r_{i}(t) \\
& =\left[\mathbf{C}_{i}+\Delta \mathbf{C}_{i}(t)\right] \delta \mathbf{x}(t)+\left[\mathbf{D}_{i}+\Delta \mathbf{D}_{i}(t)\right] \delta u(t)-\delta r_{i}(t)
\end{aligned}
$$

For steady-state control case where $\mathbf{D}_{i}+\Delta \mathbf{D}_{i}(t)=0$, there exists no adequate degree of freedom in Eq. (15) to support the regulator design. To overcome this limitation, the generalised control error presented in Eq. (14) is employed. It can be seen that $\Theta_{i}(t) \neq 0$ always holds and the real control error in Eq. (15) will be zero when the generalised one tends to zero. A complete explanation of this is presented in [8].

With the sliding surfaces in Eq. (14), we will first check whether the system in Eq. (5) has satisfactory properties after entering the sliding mode, and then establish a set of reaching laws to ensure that the sliding motions are invariant to the system uncertainties.

Once the sliding modes are achieved, i.e., $s_{i}(t)=0$, the system represented by Eq. (5) will stay on that sliding mode, and the equivalent control input for the $i-t h$ regulator can be written as

$$
\delta u_{e q}(t)=-\frac{1}{\Theta_{i}(t)} \cdot\left(\Psi_{i}(t) \delta \mathbf{x}(t)-\Gamma_{i}(t)\right)
$$

Substituting Eq. (16) into Eq. (5) yields

$$
\begin{aligned}
\delta \dot{\mathbf{x}}(t)= & {\left[\mathbf{A}+\Delta \mathbf{A}(t)-\frac{1}{\Theta_{i}(t)}(\mathbf{B}+\Delta \mathbf{B}(t)) \boldsymbol{\Psi}_{i}(t)\right] \delta \mathbf{x}(t) } \\
& +\frac{1}{\Theta_{i}(t)}(\mathbf{B}+\Delta \mathbf{B}(t)) \Gamma_{i}(t)
\end{aligned}
$$

Since $(\mathbf{B}+\Delta \mathbf{B}(t)) \Theta_{i}^{-1}(t)$ is bounded, the stability of the linear inhomogeneous Eq. (17) is equivalent to that of the corresponding homogeneous equation. Due to this, the following theorem merely requires to discuss the stability of Eq. (17) when $\Gamma_{i}(t)=0$.

Theorem 1. [14, 24, 25]Consider the linear system (17) with $\Gamma_{i}(t)=0$, then

$$
\delta \dot{\mathbf{x}}(t)=\left(\mathbf{A}-\frac{\mathbf{B} \boldsymbol{\Omega}_{i}}{\Phi_{i}}+\mathbf{N}(t)\right) \cdot \delta \mathbf{x}(t)
$$


where

$$
\begin{array}{r}
\mathbf{N}(t)=\Delta \mathbf{A}(t)+\frac{\mathbf{B} \boldsymbol{\Omega}_{i}}{\Phi_{i}}-\frac{\mathbf{B}+\Delta \mathbf{B}(t)}{\Theta_{i}(t)} \boldsymbol{\Psi}_{i}(t) \\
\Phi_{i}= \begin{cases}1 & \text { if } \mathbf{D}_{i}+\Delta \mathbf{D}_{i}(t)=0 \text { (Case 1) } \\
\mathbf{D}_{i} & \text { if } \mathbf{D}_{i}+\Delta \mathbf{D}_{i}(t) \neq 0 \text { (Case 2) }\end{cases} \\
\boldsymbol{\Omega}_{i}= \begin{cases}\mathbf{G}_{i} & \text { if } \mathbf{D}_{i}+\Delta \mathbf{D}_{i}(t)=0 \text { (Case 1) } \\
\mathbf{C}_{i} & \text { if } \mathbf{D}_{i}+\Delta \mathbf{D}_{i}(t) \neq 0 \text { (Case 2) }\end{cases}
\end{array}
$$

Suppose that for some $c_{1 i}, c_{2 i}>0$

$$
\left\|e^{\left(\mathbf{A}-\frac{\mathbf{B} \Omega_{i}}{\Phi_{i}}\right) t}\right\| \leq c_{1 i} e^{-c_{2 i} t}, \forall t \geq 0
$$

Once a set of $\sigma_{i}>0$ exist and conform to the following inequality

$$
\|\mathbf{N}(t)\| \leq \sigma_{i}<\frac{c_{2 i}}{c_{1 i}}, \forall t \geq 0, \forall i=1, \ldots, m
$$

system (18) is marginally stable for all $i$.

To guarantee the invariance properties of the SMC, a set of control laws should be generated to ensure $s_{i}(t) \cdot \dot{s}_{i}(t)<0$. Considering the following reaching laws

$$
u_{r, i}(t)=-\Phi_{i}^{-1}\left[\xi_{1 i} \cdot \rho_{i}(t) \cdot \operatorname{sgn}\left(s_{i}(t)\right)+\xi_{2 i} \cdot s_{i}(t)\right]
$$

where

$$
\rho_{i}(t)= \begin{cases}\left\|\mathbf{G}_{i} \mathbf{E}\right\| \cdot P(t)+\left|\mathbf{G}_{i}(\mathbf{A} \delta \mathbf{x}(t)+\mathbf{B} \delta u(t))\right| & \text { (Case 1) } \\ \left\|\mathbf{C}_{i} \mathbf{E}\right\| \cdot P(t)+\left|\mathbf{C}_{i}(\mathbf{A} \delta \mathbf{x}(t)+\mathbf{B} \delta u(t))\right|+k_{B} & \text { (Case 2) }\end{cases}
$$

$P(t)=\left\|\mathbf{H}_{a} \delta \mathbf{x}(t)\right\|+\left\|\mathbf{H}_{b} \delta u(t)\right\|, \xi_{1 i}>1 / \eta$, and $\xi_{2 i}>0$.

The following theorem will show the invariance of the sliding motions.

Theorem 2. Consider the system (5) operating under the $i-t h$ regulator. If the $i-t h$ reaching law is synthesised as in Eq. (24), system states can then be driven to the $i$ - th sliding surface.

Proof. Choose the Lyapunov function

$$
V(t)=\frac{1}{2} s_{i}^{2}(t)
$$

From Eqs (12) and (24), it has

$$
\begin{aligned}
\operatorname{sat}\left(u_{r, i}(t)\right) \Phi_{i} \cdot s_{i}(t) & =\omega\left(u_{r, i}(t)\right) \cdot \Phi_{i} u_{r, i}(t) \cdot s_{i}(t) \\
& =-\omega\left(u_{r, i}(t)\right)\left[\xi_{1 i} \rho_{i}(t)\left|s_{i}(t)\right|+\xi_{2 i} s_{i}^{2}(t)\right]
\end{aligned}
$$

According to Remark 3, it is obtained that

$$
\operatorname{sat}\left(u_{r, i}(t)\right) \Phi_{i} \cdot s_{i}(t)<-\eta\left[\xi_{1 i} \rho_{i}(t)\left|s_{i}(t)\right|+\xi_{2 i} s_{i}^{2}(t)\right]
$$

Case 1: $\mathbf{D}_{i}+\Delta \mathbf{D}_{i}(t)=0$

By utilising Eqs. (14) and (5), $\dot{V}(t)$ can be obtained as

$$
\begin{aligned}
\dot{V}(t)= & s_{i}(t) \cdot \dot{s}_{i}(t) \\
= & s_{i}(t) \cdot\left[\mathbf{G}_{i} \mathbf{A} \delta \mathbf{x}(t)+\mathbf{G}_{i} \mathbf{B} \delta u(t)+\operatorname{sat}\left(u_{r, i}(t)\right)\right] \\
& +s_{i}(t) \cdot\left[\mathbf{G}_{i} \Delta \mathbf{A}(t) \delta \mathbf{x}(t)+\mathbf{G}_{i} \Delta \mathbf{B}(t) \delta u(t)\right]
\end{aligned}
$$


Consider Assumption 1, then it has

$$
\begin{aligned}
\dot{V}(t) \leq & \left|s_{i}(t)\right| \cdot\left|\mathbf{G}_{i} \mathbf{A} \delta \mathbf{x}(t)+\mathbf{G}_{i} \mathbf{B} \delta u(t)\right|+s_{i}(t) \cdot \operatorname{sat}\left(u_{r, i}(t)\right) \\
& +\left|s_{i}(t)\right| \cdot\left\|\mathbf{G}_{i} \mathbf{E}\right\| \cdot\left(\left\|\mathbf{H}_{a} \delta \mathbf{x}(t)\right\|+\left\|\mathbf{H}_{b} \delta u(t)\right\|\right) \\
= & \left|s_{i}(t)\right| \cdot \rho_{i}(t)+\Phi_{i} s_{i}(t) \operatorname{sat}\left(u_{r, i}(t)\right)
\end{aligned}
$$

Combining (30) with (28), it has

$$
\begin{aligned}
\dot{V}(t) & \leq\left|s_{i}(t)\right| \cdot \rho_{i}(t)-\eta \xi_{1 i} \rho_{i}(t)\left|s_{i}(t)\right|-\eta \xi_{2 i} s_{i}^{2}(t) \\
& =\left(1-\eta \xi_{1 i}\right) \rho_{i}(t)\left|s_{i}(t)\right|-\eta \xi_{2 i} s_{i}^{2}(t)<0
\end{aligned}
$$

Case 2: $\mathbf{D}_{i}+\Delta \mathbf{D}_{i}(t) \neq 0$

By utilising Eq. (14) and (5), $\dot{V}(t)$ can be obtained as

$$
\begin{aligned}
\dot{V}(t)= & s_{i}(t) \cdot \dot{s}_{i}(t) \\
= & s_{i}(t) \cdot\left[\mathbf{C}_{i} \mathbf{A} \delta \mathbf{x}(t)+\mathbf{C}_{i} \mathbf{B} \delta u(t)+\dot{w}_{i}(t)+\mathbf{D}_{i} \operatorname{sat}\left(u_{r, i}(t)\right)\right] \\
& +s_{i}(t) \cdot\left[\mathbf{C}_{i} \Delta \mathbf{A}(t) \delta \mathbf{x}(t)+\mathbf{C}_{i} \Delta \mathbf{B}(t) \delta u(t)\right]
\end{aligned}
$$

Consider Assumptions 1 and 2, then

$$
\begin{aligned}
\dot{V}(t) \leq & \left|s_{i}(t)\right| \cdot\left(\left|\mathbf{C}_{i} \mathbf{A} \delta \mathbf{x}(t)+\mathbf{C}_{i} \mathbf{B} \delta u(t)\right|+k_{B}\right)+\mathbf{D}_{i} s_{i}(t) \operatorname{sat}\left(u_{r, i}(t)\right) \\
& +\left|s_{i}(t)\right| \cdot\left\|\mathbf{C}_{i} \mathbf{E}\right\| \cdot\left(\left\|\mathbf{H}_{a} \delta \mathbf{x}(t)\right\|+\left\|\mathbf{H}_{b} \delta u(t)\right\|\right) \\
= & \left|s_{i}(t)\right| \cdot \rho_{i}(t)+\Phi_{i} s_{i}(t) \operatorname{sat}\left(u_{r, i}(t)\right)
\end{aligned}
$$

Combining (33) with (28), then

$$
\begin{aligned}
\dot{V}(t) & \leq\left|s_{i}(t)\right| \cdot \rho_{i}(t)-\eta \xi_{1 i} \rho_{i}(t)\left|s_{i}(t)\right|-\eta \xi_{2 i} s_{i}^{2}(t) \\
& =\left(1-\eta \xi_{1 i}\right) \rho_{i}(t)\left|s_{i}(t)\right|-\eta \xi_{2 i} s_{i}^{2}(t)<0
\end{aligned}
$$

So far, the invariance of the sliding motions has been proved, i.e., employing the reaching laws in Eq. (24) can ensure that the system states globally converge to the corresponding sliding surface in finite time despite the existence of the rate constraints and the uncertainties.

\subsection{Additional discussions}

Among various kinds of practical issues in aircraft engine control, some crucial considerations are discussed in the following subsections.

\subsubsection{Entire switching system}

According to the switching framework as shown in Fig. 1, all the regulators can be divided into two sets, Sets $L$ and $H$. The regulators connected to the min selector are all included in Set $L$, while the rests that connected to the max selector are included in Set $H$. With the definition of $L$ and $H, q_{\min }$ and $q_{\max }$ are used to denote the indices of the selected regulators.

$$
\begin{gathered}
q_{\min }(t)=\arg \min _{j \in L}\left\{u_{r, j}(t)\right\} \\
q_{\max }(t)=\arg \max _{k \in H}\left\{u_{r, k}(t)\right\}
\end{gathered}
$$


where subscript 'min' and 'max' for the min and max selector respectively. Accordingly, the overall regulator index $q$ can be expressed as

$$
q(t)= \begin{cases}q_{\text {min }}(t) & \text { if } \min _{j \in L}\left\{u_{r, j}(t)\right\} \geq \max _{k \in H}\left\{u_{r, k}(t)\right\} \\ q_{\text {max }}(t) & \text { if } \min _{j \in L}\left\{u_{r, j}(t)\right\}<\max _{k \in H}\left\{u_{r, k}(t)\right\}\end{cases}
$$

With the aid of $q$, the overall control input and the overall sliding function can be expressed as $u_{r}(t)=$ $u_{r, i=q}(t)$ and $s(t)=s_{i=q}(t)$ respectively.

Even though the system can be stabilised by every single regulator, it cannot guarantee that the entire switching system is stable. Multiple Lyapunov approaches from [26, 27] have been proposed for the switching problems, however, it is difficult to find an appropriate Lyapunov function or to meet some certain requirements.

Applying the theory of invariant set is a solution to overcome this difficulty. Ritcher[6] shows a global stability of a multi-regulator system switched by max-min selectors. In the case of min switching, for example, he firstly proved that when $s(t)=0$ and $i=q$, the set of the states is invariant. And then, he proved that when $i=q, s(t)$ tends to be zero in finite time. Finally, he showed that for $i \neq q$, index $i$ switches to $q$ in finite time. The results of his paper explain the stability of our switching system after some modifications.

\subsubsection{Measurement noise}

Consider that a bounded noise $\mu_{n}(t)$ is introduced to measurement. In such a practical case, the measured $s_{i}$, says $\hat{s}_{i}$, will have a different value from the real one due to the noise, i.e.

$$
\hat{s}_{i}(t)=s_{i}(t)+\mu_{n}(t) \quad\left|\mu_{n}(t)\right| \leq \delta
$$

According to which the sliding surface $s_{i}(t)=0$ is replaced by a sliding layer $\hat{s}_{i}(t) \leq \delta$. For the sliding layer, Theorem 1 can be adopted without any change because $s_{i}(t)$ is assumed to be zero when $\hat{s}_{i}(t) \leq \delta$. As for the reaching laws, Eq. (24) should be supplanted by

$$
u_{r, i}(t)=-\Phi_{i}^{-1}\left[\xi_{1 i} \cdot \hat{\rho}_{i}(t) \cdot \operatorname{sgn}\left(\hat{s}_{i}(t)\right)+\xi_{2 i} \cdot \hat{s}_{i}(t)\right]
$$

Compared to $\rho_{i}$ in Eq. (25), $\hat{\rho}_{i}$ in Eq. (38) should include an additional gain to compensate for the bounded noise. By applying Theorem 2 with some modifications, however, it is not difficult to prove that Eq. (38) can drive the system state trajectories into the $i-t h$ sliding layer $\hat{s}_{i}(t) \leq \delta$.

\subsubsection{External disturbance}

For an aircraft engine, the change of environmental parameters is typically regarded as a major external disturbance. Even though SMC method is suitable for solving problems with disturbance, applying it to a single design still cannot cover the entire flight envelope with satisfactory performance. Because ambient conditions such as temperature and pressure vary in a considerably wide range. To address the entire envelope, a piecewise linear method is commonly used to divide the whole range into small pieces. Add a disturbance $\mu_{d}(t)$ to Eq. (4)

$$
\delta \dot{\mathbf{x}}(t)=\mathbf{A} \cdot \delta \mathbf{x}(t)+\mathbf{B} \cdot \delta u(t)+\mu_{d}(t)
$$

Within a small area, the environmental disturbance is small enough and can be converted into the model uncertainties, i.e., $\mu_{d}(t)=\Delta \mathbf{A}^{\prime}(t) \delta \mathbf{x}(t)+\Delta \mathbf{B}^{\prime}(t) \delta u(t)$. As a result, Eq. (5) can be employed directly to represent the disturbed system where $\Delta \mathbf{A}$ and $\Delta \mathbf{B}$ are presumed to contain $\Delta \mathbf{A}^{\prime}$ and $\Delta \mathbf{B}^{\prime}$ respectively.

\section{Application to turbofan engine}

The presented method is applied to a model turbofan similar to the General Electric CF6 series. The model was created utilising library GTML-E developed on behalf of Beihang University. GTML-E is an 
open source MATLAB/Simulink library for gas turbine modeling and simulation[28]. The Iterative Newton Raphson Solver block used in GTML-E is based on T-MATS package[19]. All component characteristic maps and relevant data are acquired from a commercial software called GSP[29]. The employed model is quite mature and has been applied to other researches on control design [14, 23, 30].

\subsection{Preparation for numerical simulation}

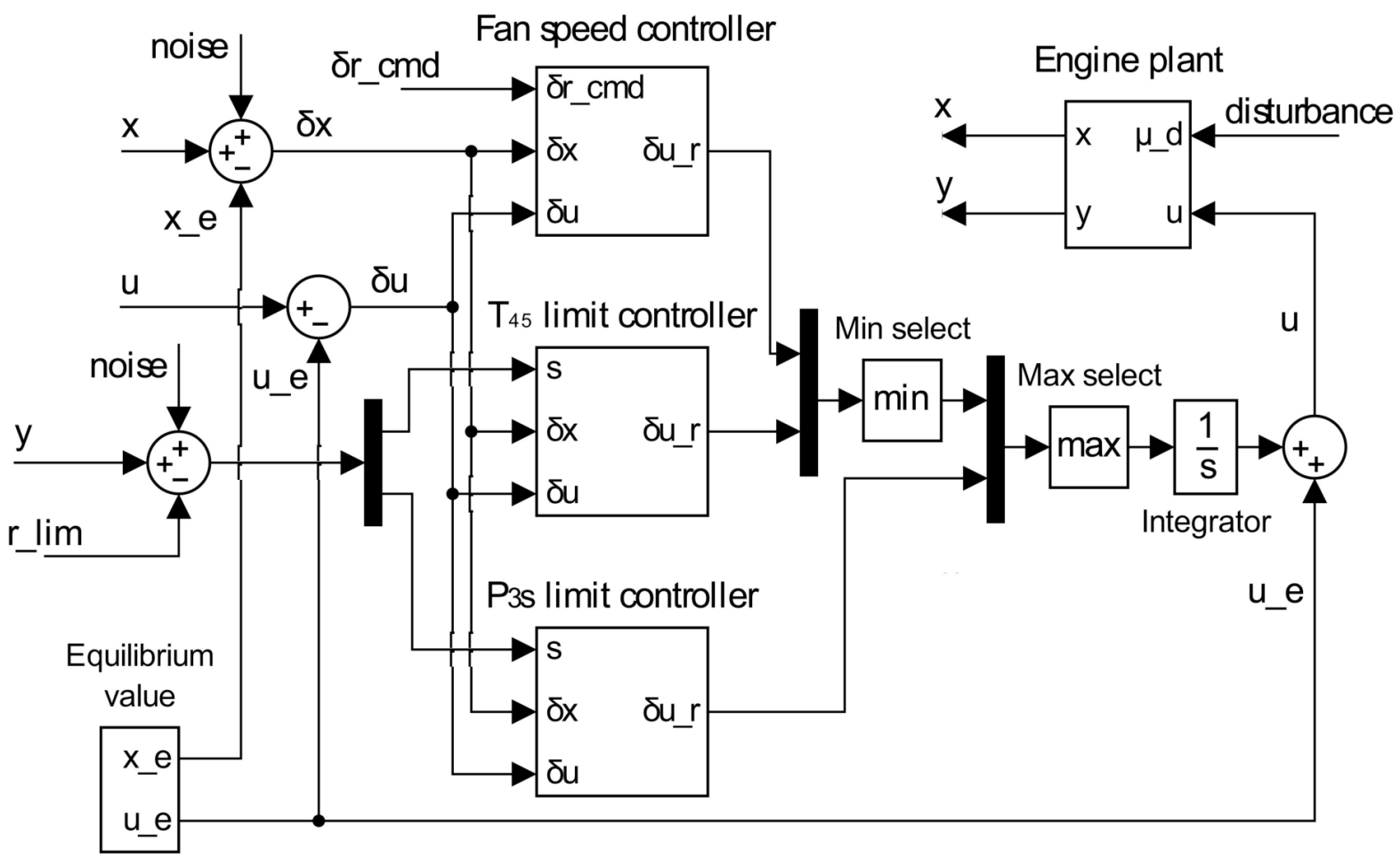

Figure 2: Simulation architecture of the closed-loop system.

Fig. 2 exhibits the simulation architecture of the closed-loop system, which consists of the model turbofan engine and a set of controllers within the max-min selection framework. The plant has a single input, three outputs, and two state parameters. It should be noted that all above parameters have been normalised with their nominal values: control input $u(t)$ is the normalised fuel flow $\left(W_{f}(t) / W_{f, n}\right)$; outputs $y_{1}(t)$, $y_{2}(t)$, and $y_{3}(t)$ are the normalised exhaust gas temperature, high-pressure compressor outlet pressure, and fan speed respectively $\left(T_{45}(t) / T_{45, n}, P_{3 s}(t) / P_{3 s, n}\right.$, and $\left.N_{1}(t) / N_{1, n}\right)$. In addition, $x_{1}(t)$ and $x_{2}(t)$ are the normalised fan and core speed $\left(N_{1}(t) / N_{1, n}\right.$ and $\left.N_{2}(t) / N_{2, n}\right)$.

For a standard day under the cruise condition, Tab. 2 summarises nominal and limit values of above variables in this application. At the nominal point, the engine model is linearised to obtain the linearisation matrices as follows

$$
\begin{aligned}
\mathbf{A}=\left[\begin{array}{cc}
-2.4797 & 0.8963 \\
-0.3582 & -1.0845
\end{array}\right], \mathbf{B}=\left[\begin{array}{c}
0.4881 \\
0.2845
\end{array}\right. \\
\mathbf{C}=\left[\begin{array}{cc}
-0.5696 & -0.0446 \\
1.2149 & 0.8238 \\
1.0 & 0.0
\end{array}\right], \mathbf{D}=\left[\begin{array}{c}
0.4848 \\
0.2028 \\
0
\end{array}\right]
\end{aligned}
$$


Table 2: Turbofan nominal parameters of cruise (Altitude 10,668 m, Mach Number 0.84)

\begin{tabular}{clll}
\hline Parameter & Value & Unit & Limit \\
\hline$W_{f}$ & 0.85 & $\mathrm{~kg} / \mathrm{s}$ & - \\
$N_{1}(t)$ & 3,180 & $\mathrm{rpm}$ & - \\
$N_{2}(t)$ & 9,618 & $\mathrm{rpm}$ & - \\
$T_{45}$ & 944.2 & $\mathrm{~K}$ & $\leq 1,050$ \\
$P_{3 s}$ & 1,123 & $\mathrm{kPa}$ & $\geq 780$ \\
\hline
\end{tabular}

\subsection{Limitation of existing researches}

As for the sliding mode strategy, especially when mechanical actuators are involved, most of the researchers focus on its high-frequency nature, which can lead to chattering issues. However, only attenuating the chattering is far from satisfactory. Even if the chattering phenomenon can be effectively attenuated by no matter what methods, system constraints may lead to severe degradation of system performance. In this subsection, three types of system constraints are discussed and the adverse effect of the constraints is revealed.

a) Input constraints:

When a border of input constraints is reached, some control inputs will be saturated and therefore bring static tracking errors to the closed-loop system. Fortunately, this doesn't affect the system stability as discussed in Remark 1.

b) Input rate constraints:

Classical SMC methods often conflict with input rate constraints due to its high-frequency switching nature. Once the realistic input rate cannot follow the desired value, the condition of Lyapunov stability will be violated. As a result, the closed-loop system may oscillate or even become unstable.

c) Output constraints:

Unlike the other two types of constraint, output constraints are introduced on designers' own initiative. By using the max-min switching framework, output constraints are designed to safeguard an engine from operating under overload.

Since rate constraint is a major concern, two simulations are carried out to show its adverse effect. The existing SMC design from [6] is applied in both simulations. In the first one, an ideal situation is considered where the input rate of the plant can be infinitely large. In the other one, a practical situation is considered where the input rate is limited by $k_{A}=0.5\left(\mathrm{~kg} / \mathrm{s}^{2}\right)$. In both situations, the engine is firstly accelerated from an equilibrium point to another one with a $280(\mathrm{rpm})$ increment of fan speed. After 4 seconds, the engine is decelerated back to the original equilibrium point.

Fig. 3 shows the trajectories of fan speed $N_{1}$ and exhaust gas temperature $T_{45}$ under the two situations. In the ideal situation, $N_{1}$ has smooth responses while $T_{45}$ maintains its value below the limit of 1050 (K). In the rate limited situation, however, $N_{1}$ responds unsmoothly, $T_{45}$ exceeds the limit and oscillates considerably.

Fig. 4 shows the trajectories of overall control input rate $u_{r}$ and the overall sliding function $s$ under two different situations. In the ideal case, unconstrained $u_{r}$ guarantees that $s$ converges monotonously. As for the rate limited case, $u_{r}$ is bounded within its limit value $k_{A}=0.5\left(\mathrm{~kg} / \mathrm{s}^{2}\right)$ but $s$ can no longer converge to zero monotonously. The occasional jumps of the sliding function $s(t)$ violate the monotonous reaching property, which is the cause of the noticeable temperature oscillation in Fig. 3. With a proper set of control gains, no chattering phenomenon is shown in Figs. 3 and 4 . In this case, however, Fig. 4 shows that the rate constraints can still cause the closed-loop system oscillating severely.

According to the results, it can be concluded that merely attenuating the chattering, which has been extensively studied by the researchers, has limited ability to improve the closed-loop performance. 

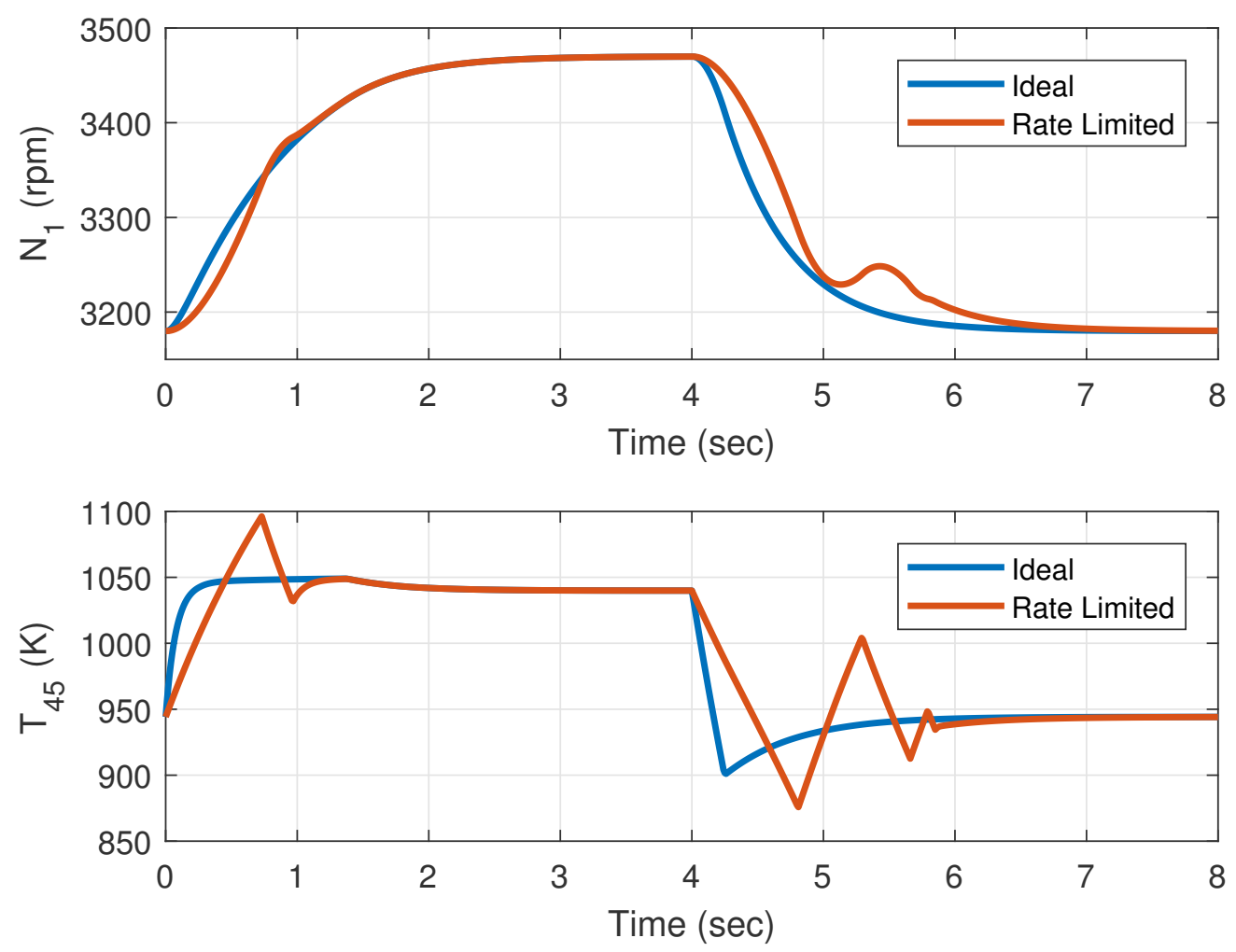

Figure 3: Influence of input rate constraints on the classical SMC design. Compared to the ideal case, the rate limited case shows unsmooth $N_{1}$ (fan speed), overshot and oscillated $T_{45}$ (exhaust gas temperature).

\subsection{Parameter selection for the design}

The parameter selection of the presented design can be divided into three steps. The first step is to determine a desired dynamic as reference. As mentioned before, $\mathbf{G}$ is introduced as an extra degree of design freedom, which gives us the opportunity to choose a desired pole of the nominal dynamics $\mathbf{A}-\mathbf{B G}$. In this application case, for example, once the pole $(-2.2,-1.6)$ is decided, $\mathbf{G}$ can be obtained as $\mathbf{G}=\left[\begin{array}{ll}-0.55 & 1.78\end{array}\right]$ by pole placement. The second step is to choose the reaching law gains $\xi$. According to the proposed reaching laws, the gains should satisfy $\xi_{1 i}>1 / \eta$ and $\xi_{2 i}>0$, where $i=1, \ldots, m$. Generally, a larger gain may increase the reaching rate but an excessively large gain can cause undesirable chattering phenomenon. As a result, a smaller gain that still leads to a satisfactory reaching rate is preferred out of safety concerns. The third step is to determine the parameter values of $\mathbf{H}_{a}, \mathbf{H}_{b}, \mathbf{E}$, and $k_{B}$, which are related to the ranges of uncertainties. It should be noted that only when the ranges are wide enough to cover the actual model mismatch (Assumptions 1 and 2 are satisfied) can Theorems 1 and 2 guarantee a robust control design. In practice, however, trial and error may be involved in the second or the third step of the selection. The final selection result of the presented design is shown as in Eq. (41).

$$
\begin{gathered}
k_{B}=0.06, \mathbf{H}_{b}=\left[\begin{array}{ll}
0.2 & 0.2
\end{array}\right]^{T} \\
\mathbf{H}_{a}=\left[\begin{array}{cc}
0.5 & 0 \\
0 & 0.5
\end{array}\right], \mathbf{E}=\left[\begin{array}{ll}
1 & 0 \\
0 & 1
\end{array}\right], \xi=\left[\begin{array}{ccc}
2.6 & 2.6 & 2.6 \\
50 & 25 & 10
\end{array}\right]
\end{gathered}
$$

The gains in $\xi$ are vital parameters to be adjusted. To show the influence of different gains, a comparative study is carried out as follows. Besides the ideal case and the rate limited case with $\xi$, two other simulations are conducted with different gains, e.g., $0.5 \xi$ and $2 \xi$. All simulations are carried out with the engine firstly accelerating from the cruise point to the other point and then decelerating back to its starting condition. 

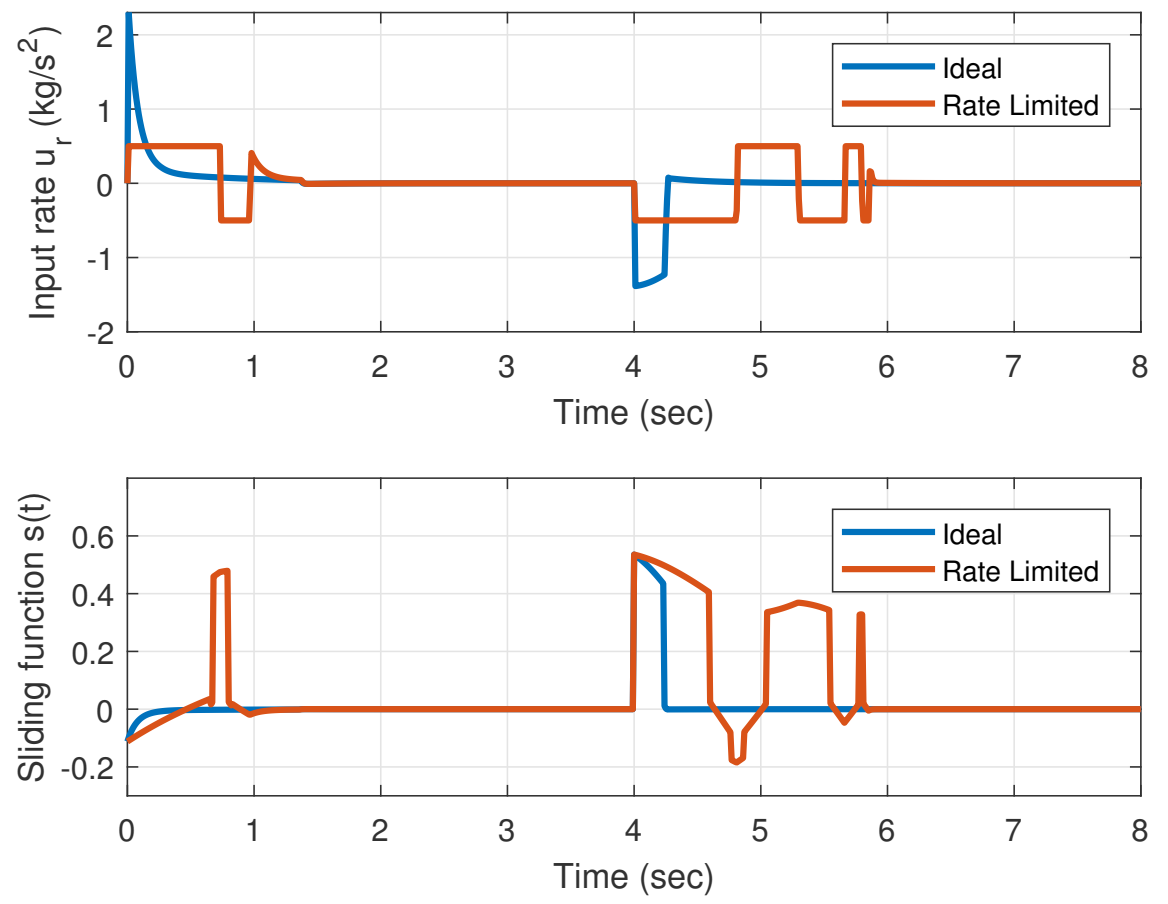

Figure 4: Limited $u_{r}$ (overall control input rate) and its influence on $s$ (overall sliding function). The ideal case shows that $u_{r}$ can be large enough to guarantee the $s$ with monotone convergence. While the rate limited case shows the $s$ with non-monotone convergence.

Fig. 5 shows the trajectories of fan speed $N_{1}$ and exhaust gas temperature $T_{45}$ under the four cases. Compared with the ideal case, all the rate limited cases show slower responses. For every case, $N_{1}$ has a smooth response and $T_{45}$ maintains its value below the limit of $1050(\mathrm{~K})$. In the scaled footage of $N_{1}$, the response in rate limited case with $\xi$ (yellow) is obviously higher than that with $0.5 \xi$ (violet) but slightly lower than that with $2 \xi$ (orange). This shows that increasing the gains can lead to a faster response. However, the increase in response speed has an upper bound because of the rate constraints. A similar situation can be found in the footage of $T_{45}$ where larger gains result in faster responses.

Fig. 6 shows the trajectories of overall control input rate $u_{r}(t)$ and the overall sliding function $s(t)$ under the four cases. The ideal case shows an unconstrained $u_{r}(t)$ while the rate limited cases show the $u_{r}(t)$ bounded by its limit value $k_{A}=0.5\left(\mathrm{~kg} / \mathrm{s}^{2}\right)$. All cases show that $s(t)$ converges to zero monotonously. In the left footage of $u_{r}(t)$, the rate constraint can be recognised as a straight line above the $u_{r}(t)$ in all rate limited cases. Since larger gains mean faster responses of the engine, it is not surprising that $u_{r}(t)$ with $2 \xi$ stays on its constraint for the longest time among the three rate limited cases. Although a faster response for better performance is always preferred, the gains should not be excessively large because of its unacceptable chattering. In the right footage of $u_{r}(t)$, the case with $0.5 \xi$ shows no chattering(violet), the standard case with $\xi$ shows moderate chattering(yellow) while the case with $2 \xi$ shows the worst chattering(orange). Actually, it is crucial to decide appropriate gains for the control laws. In practice, the trade-off between performance(response speed) and safety(chattering) should always be taken into consideration.

\subsection{Against disturbance and noise}

A final simulation is carried out with the parameters selected above. To show the abilities of noise attenuation and disturbance rejection, noise and disturbance are introduced in the simulation as shown in Fig. 2. Disturbance is represented by the change of environmental parameters. Therefore, the constant values (Altitude 10,668 m, Mach Number 0.84) can be replaced by time-dependent functions, e.g., Altitude = 

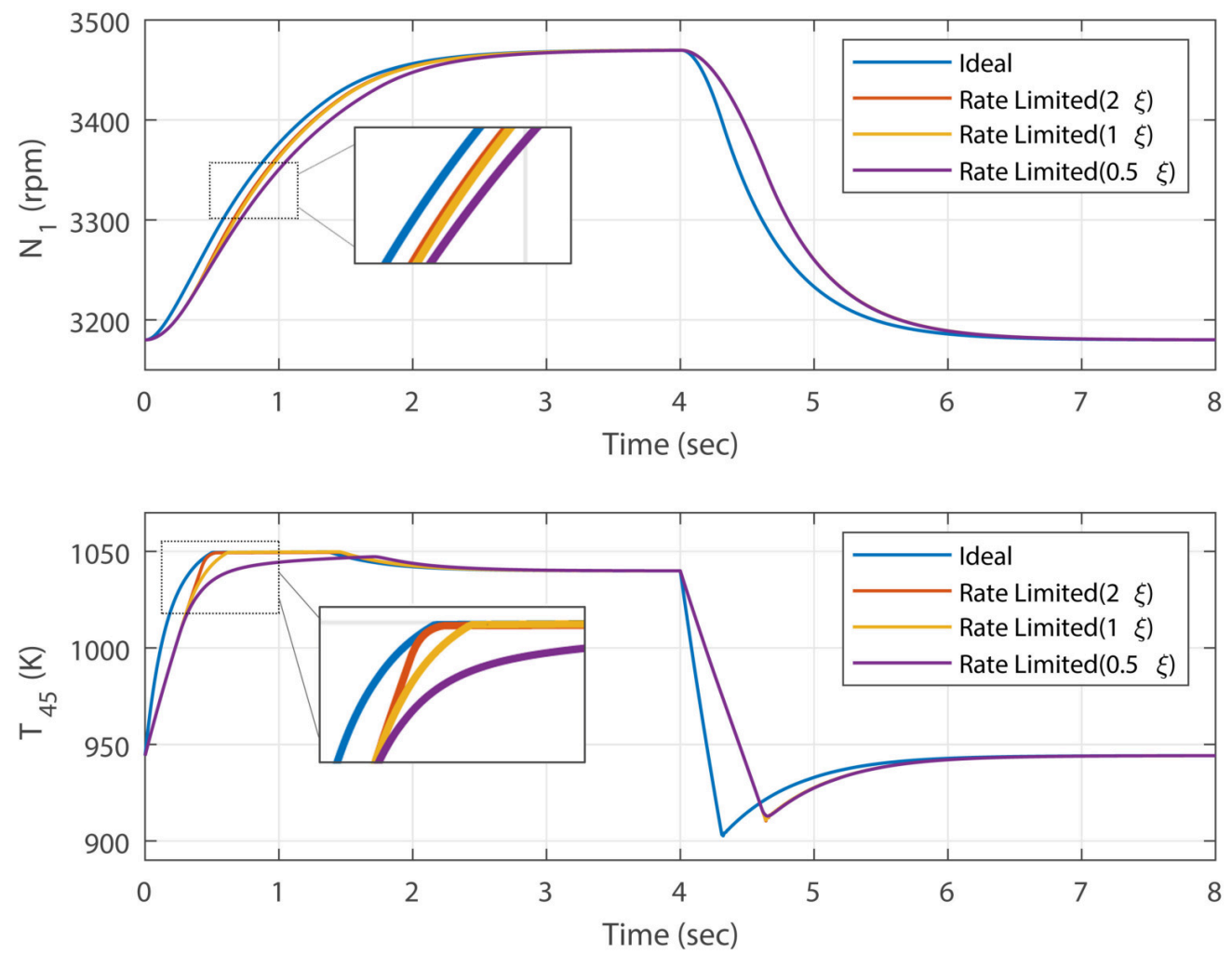

Figure 5: Fan speed and exhaust gas temperature responses of proposed SMC design. Compared to the ideal case, the rate limited cases shows slower responses. All cases show smooth $N_{1}$ and bounded $T_{45}$.

$10668+15 t$ and Mach Number $=0.84+0.001 t$. Bounded noise $\mu_{n}(t)$ with $\delta=0.01$ is added to all the normalised measurements $x_{1}, x_{2}, y_{1}, y_{2}$, and $y_{3}$.

Fig. 7 shows the trajectories of $N_{1}, T_{45}, s$, and $W_{f}$. The plots of $N_{1}$ and $T_{45}$ are similar to their counterparts in Fig. 5 but contain obvious measurement noise. Although the disturbance of environmental parameters is added to the simulation, $s$ converges to zero monotonously, indicating that the proposed design has the ability of disturbance rejection during tracking and protecting process. In spite of the rough measurements, the output of control $W_{f}$ exhibits an almost smooth result, which proves noise attenuation ability of the design.

Fig. 8 exhibits the transient operating lines on different component maps. The model turbofan accelerates from the design point to another equilibrium point in the first 4 seconds, and then decelerates back to the design point, leaving sufficient surge margin on the maps.

With neither iteration nor searching process involved, the calculation of the proposed control law is relatively direct and simple. Specifically, once engine states $\mathbf{x}$ and outputs $\mathbf{y}$ are received, each regulator can calculate its corresponding control input rate $u_{r, i}$ according to Eq. (24). Afterward, the max-min select logic will decide which regulator is active and the downstream integrator will generate the actual control input $u$. Due to such a simple calculation, the computing speed is quite fast. By using a personal computer with a $1.8 \mathrm{GHz}$ processor, for instance, it took about 5 seconds to finish the 8 seconds simulation where the sample time is 20 milliseconds and even the calculation of the model engine is included. Consequently, there should be no worry about the real-time computing performance of the proposed control scheme. 

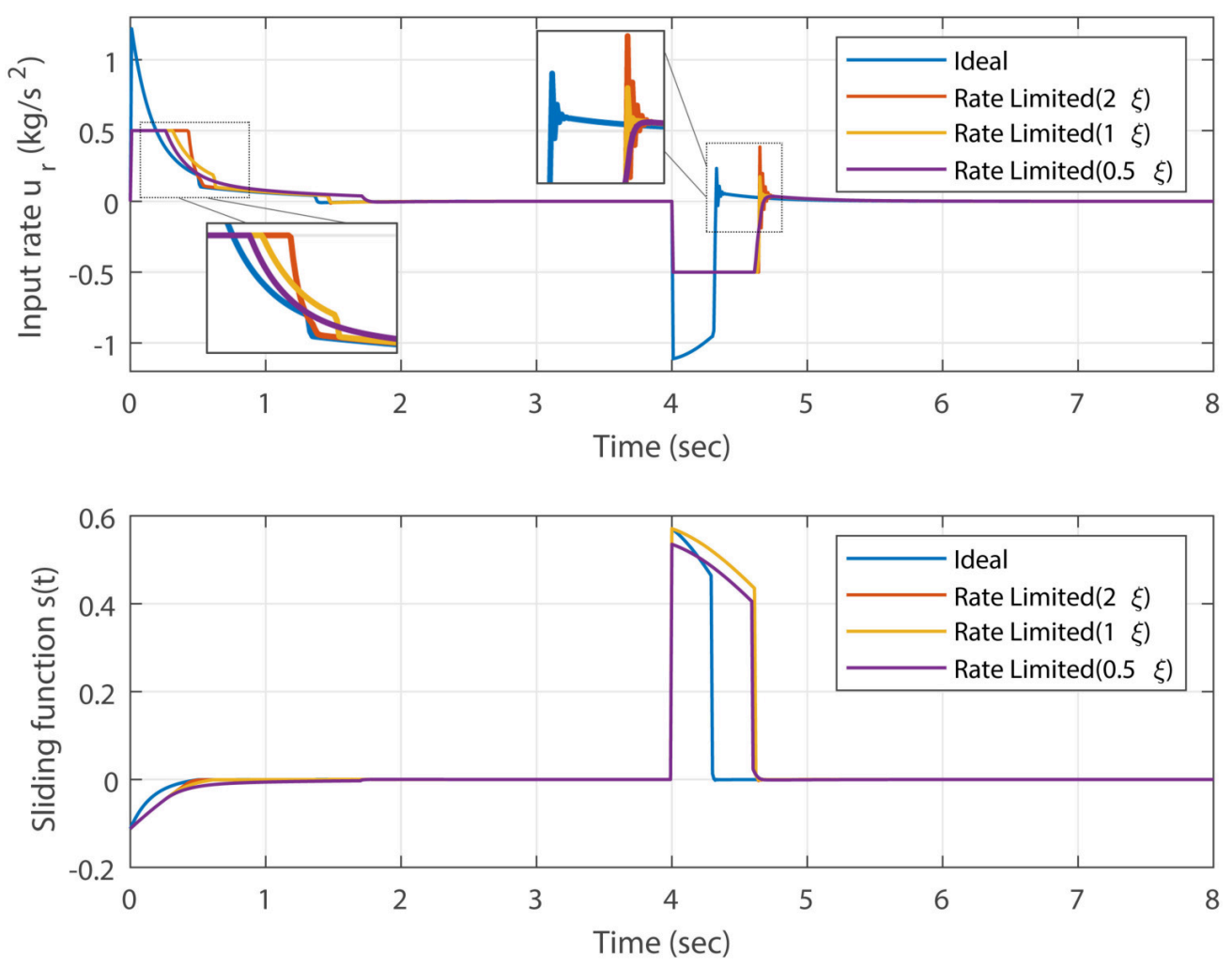

Figure 6: Overall control input rate $u_{r}$ and sliding function $s$. The ideal case and the rate limited cases show unconstrained and constrained $u_{r}$ respectively. All cases show that $s$ converges monotonously.

\section{Conclusions}

For aircraft engines, it is important to consider system constraints on input, output, and input rate when the control system is designed. Tab. 3 briefs us the discussion on different types of constraints. Among the three types of constraints, special attention should be paid to input rate constraints because they may result in oscillating and even unstable responses of the closed-loop system. In addition, if the input rate constraints are not considered in practice, SMC with discontinuous laws will have no engineering significance in the aircraft engine control.

Under the constraints, the design of SMC is discussed for both main regulators (case $\mathbf{D}=0$ ) and limit regulators (case $\mathbf{D} \neq 0$ ). With a set of sliding surfaces defined, a set of SMC laws with global stability analysis are proposed. Case studies show that, compared to the existing designs, the proposed one has good tracking and protecting capability under the system constraints. Moreover, the design has proven abilities of noise attenuation and disturbance rejection.

The proposed method is valid for a single-input system and is merely in the neighborhood of an equilibrium point. Multi-input systems and large-scale analysis should be considered in future research.

\section{References}

[1] L. Jaw, J. Mattingly, Aircraft Engine Controls, American Institute of Aeronautics and Astronautics, Inc., Washington, DC, 2009. doi:10.2514/4.867057.

URL http://arc.aiaa.org/doi/book/10.2514/4.867057

[2] V. I. Utkin, Survey Paper: Variable Structure Systems with Sliding Modes, IEEE Transactions on Automatic Control 22 (2) (1977) 212-222. doi:10.1109/TAC.1977.1101446.

[3] H. R. Karimi, Y. Zou, Y. Liu, Y. Niu, Adaptive sliding mode reliable control for switched systems with actuator degradation, IET Control Theory \& Applications 9 (8) (2015) 1197-1204. doi:10.1049/iet-cta.2014.0419. 

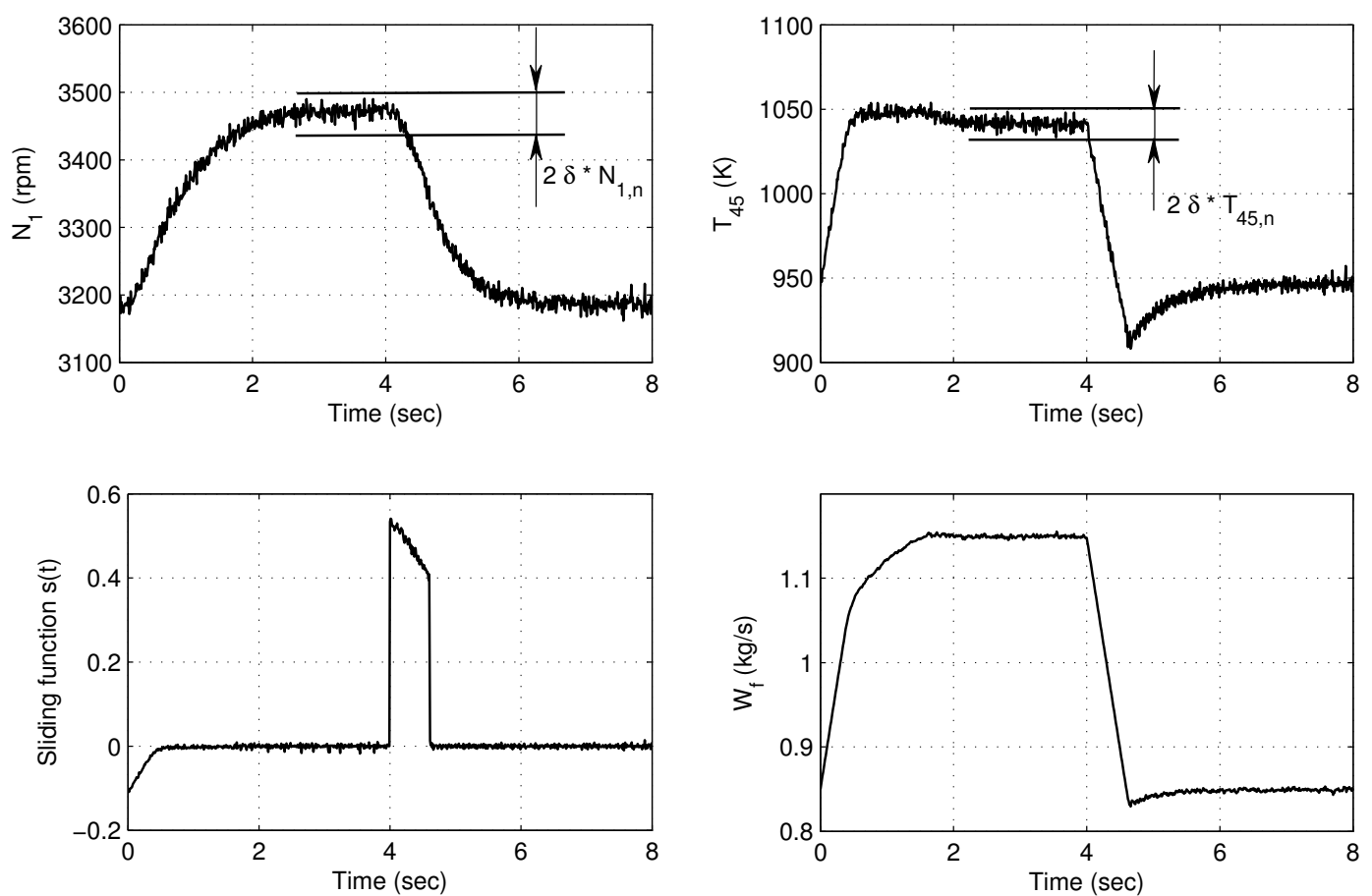

Figure 7: Fan speed, exhaust gas temperature, sliding function, and fuel flow mass responses in the final simulation.

Table 3: Discussion on different types of constraints for aircraft engines

\begin{tabular}{clll}
\hline Type & Origin & Consequence of neglect & Suggestion for treatment \\
\hline Input & Exist in practice & Static tracking error & Limit the input directly \\
Output & Introduced artificially & Exceed the limits & The switching framework \\
Input rate & Exist in practice & Unstable behave & Consider in design process \\
\hline
\end{tabular}

[4] S. Panda, B. Bandyopadhyay, Sliding Mode Control of Gas Turbines Using Multirate-Output Feedback, Journal of Engineering for Gas Turbines and Power 130 (3) (2008) 034501. doi:10.1115/1.2830546.

[5] M. Roopaei, M. Zolghadri Jahromi, Chattering-free fuzzy sliding mode control in MIMO uncertain systems, Nonlinear Analysis, Theory, Methods and Applications 71 (10) (2009) 4430-4437. doi:10.1016/j.na.2009.02.132.

[6] H. Richter, A multi-regulator sliding mode control strategy for output-constrained systems, Automatica 47 (10) (2011) 2251-2259. doi:10.1016/j.automatica.2011.08.003.

[7] X. Du, H. Richter, Y. Guo, Multivariable Sliding-Mode Strategy with Output Constraints for Aeroengine Propulsion Control, Journal of Guidance, Control, and Dynamics 39 (7) (2016) 1631-1642. doi:10.2514/1.g001802.

[8] H. Richter, Advanced Control of Turbofan Engines, Vol. 9781461411, Springer New York, New York, NY, 2012, pp. 1-266. doi:10.1007/978-1-4614-1171-0. URL http://link.springer.com/10.1007/978-1-4614-1171-0

[9] L. Chen, C. Edwards, H. Alwi, Integral sliding mode fault-tolerant control allocation for a class of affine nonlinear system, International Journal of Robust and Nonlinear Control 29 (3) (2019) 565-582. doi:10.1002/rnc.4282.

[10] H. Du, X. Yu, M. Z. Chen, S. Li, Chattering-free discrete-time sliding mode control, Automatica 68 (2016) 87-91. doi:10.1016/j.automatica.2016.01.047.

[11] C. Zheng, J. Zhang, Finite-Time Nonlinear Disturbance Observer Based Discretized Integral Sliding Mode Control for PMSM Drives, Journal of Power Electronics 18 (4) (2018) 1075-1085. doi:10.6113/JPE.2018.18.4.1075.

[12] J. Wang, Z. Gao, Y. Fu, Chattering-free discrete-time sliding mode control with event-trigger strategy, in: Pro- 

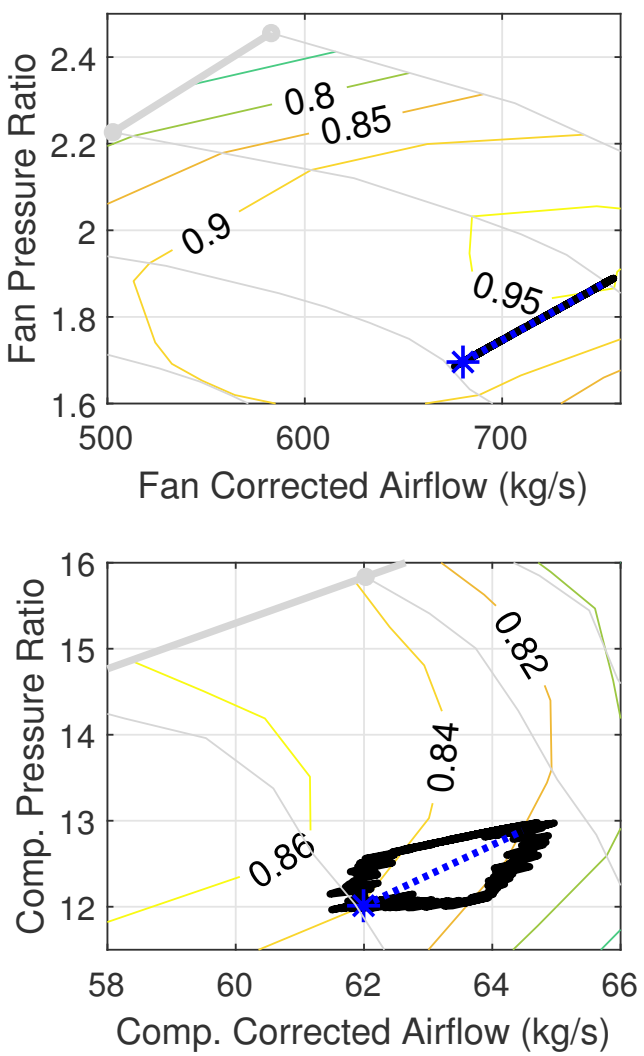
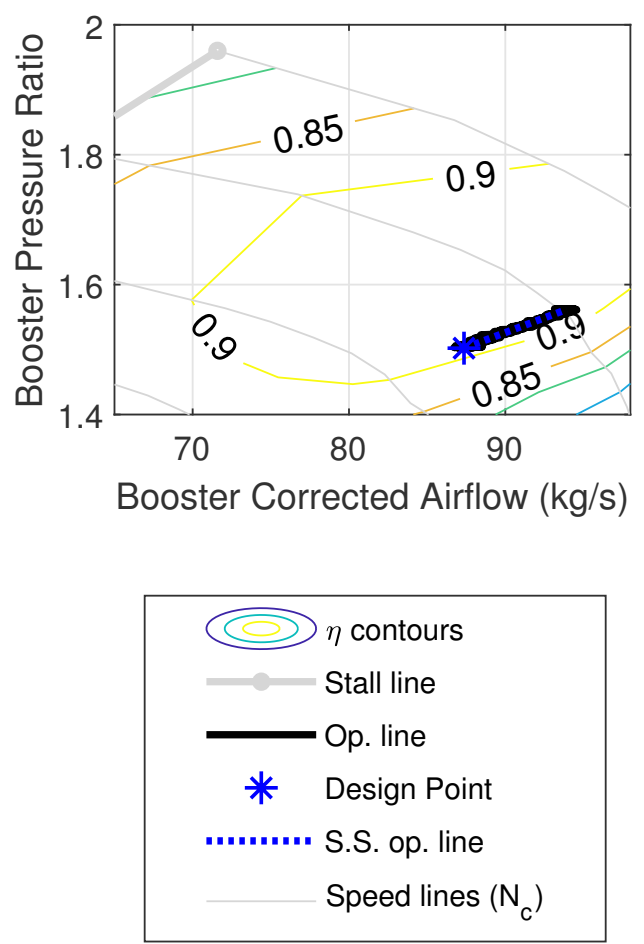

Figure 8: Operating lines on fan, booster, and compressor maps. Both acceleration and deceleration have sufficient surge margin.

ceedings - IEEE 16th International Conference on Industrial Informatics, INDIN 2018, IEEE, 2018, pp. 629-634. doi:10.1109/INDIN.2018.8471923.

[13] H. Ma, J. Wu, Z. Xiong, A novel exponential reaching law of discrete-time sliding-mode control, IEEE Transactions on Industrial Electronics 64 (5) (2017) 3840-3850. doi:10.1109/TIE.2017.2652390.

[14] S. Yang, X. Wang, B. Yang, Adaptive sliding mode control for limit protection of aircraft engines, Chinese Journal of Aeronautics 31 (7) (2018) 1480-1488. doi:10.1016/j.cja.2018.05.011.

[15] J. Na, X. Ren, G. Herrmann, Z. Qiao, Adaptive neural dynamic surface control for servo systems with unknown dead-zone, Control Engineering Practice 19 (11) (2011) 1328-1343. doi:10.1016/j.conengprac.2011.07.005.

[16] F. L. Lewis, W. K. Tim, L. Z. Wang, Z. X. Li, Deadzone compensation in motion control systems using adaptive fuzzy logic control, IEEE Transactions on Control Systems Technology 7 (6) (1999) 731-742. doi:10.1109/87.799674.

[17] M. Pakmehr, N. Fitzgerald, E. M. Feron, J. S. Shamma, A. Behbahani, Gain Scheduled Control of Gas Turbine Engines: Stability and Verification, Journal of Engineering for Gas Turbines and Power 136 (3) (2013) 031201. doi: $10.1115 / 1.4025637$.

[18] J. DeCastro, J. Litt, D. Frederick, A Modular Aero-Propulsion System Simulation of a Large Commercial Aircraft Engine, in: 44th AIAA/ASME/SAE/ASEE Joint Propulsion Conference \& Exhibit, American Institute of Aeronautics and Astronautics, Reston, Virigina, 2008. doi:10.2514/6.2008-4579.

[19] J. W. Chapman, T. M. Lavelle, R. May, J. S. Litt, T.-H. Guo, Propulsion System Simulation Using the Toolbox for the Modeling and Analysis of Thermodynamic Systems (T-MATS), in: 50th AIAA/ASME/SAE/ASEE Joint Propulsion Conference, American Institute of Aeronautics and Astronautics, Reston, Virginia, 2014. doi:10.2514/6.2014-3929.

[20] J. C. Chin, J. T. Csank, W. J. Haller, J. A. Seidel, An Introduction to Transient Engine Applications Using the Numerical Propulsion System Simulation (NPSS) and MATLAB @, Tech. Rep. January 2016, NASA Glenn Research Center (2016). URL http://www.sti.nasa.gov

[21] J. Lam, Z. Shu, S. Xu, E. K. Boukas, Robust H-infinity control of descriptor discrete-time Markovian jump systems, International Journal of Control 80 (3) (2007) 374-385. doi:10.1080/00207170600999322.

[22] P. Shi, E. K. Boukas, R. K. Agarwal, Control of Markovian jump discrete-time systems with norm bounded uncertainty and unknown delay, IEEE Transactions on Automatic Control 44 (11) (1999) 2139-2144. doi:10.1109/9.802932.

[23] S. Yang, X. Wang, Sliding Controller Design for Aero-Engines With the Rate Limitation of Actuators, in: Proceedings of the ASME Turbo Expo, Vol. 6, 2017, p. V006T05A009. doi:10.1115/gt2017-63571. 
[24] J. Shamma, Analysis and design of gain scheduled control systems, Ph.D. thesis, Massachusetts Institute of Technology (1988).

[25] R. Bellman, Stability Theory of Differential Equations, Courier Corporation, 2008.

[26] M. S. Branicky, Multiple Lyapunov functions and other analysis tools for switched and hybrid systems, IEEE Transactions on Automatic Control 43 (4) (1998) 475-482. doi:10.1109/9.664150.

[27] J. Zhao, D. J. Hill, On stability, 12-gain and h-infinity control for switched systems., Automatica 44 (5) (2008) $1220-1232$. doi:10.1016/j.automatica.2007.10.011.

[28] S. Yang, GTML-E: Gas Turbine Modeling Library for Education (2019). doi:10.5281/zenodo.2588802.

[29] W. P. J. Visser, M. J. Broomhead, GSP, a Generic Object-Oriented Gas Turbine Simulation Environment, in: Volume 1: Aircraft Engine; Marine; Turbomachinery; Microturbines and Small Turbomachinery, American Society of Mechanical Engineers, ASME, 2000, p. V001T01A002. doi:10.1115/2000-GT-0002.

[30] S. Yang, X. Wang, A Comparative Study on N-Dot Acceleration Technique, in: Proceedings of the ASME Turbo Expo, Vol. 6, 2016, p. V006T05A018. doi:10.1115/gt2016-57341. 
2019-09-19

\section{Sliding mode control with system constraints for aircraft engines}

Yang, Shu-Bo

Elsevier

Yang S-B, Wang X, Wang H-N, et al., (2020) Sliding mode control with system constraints for aircraft engines. ISA Transactions, Volume 98, March 2020, pp. 1-10

https://doi.org/10.1016/j.isatra.2019.08.020

Downloaded from Cranfield Library Services E-Repository 